\title{
Successful management of a patient with massive vocal fold granuloma with transglottal jet ventilation
}

\author{
Jieun Kim, Jong-Min Sun, Chang Kyun Kim, Nar Hyun Min, and Wyun Kon Park
}

Department of Anesthesiology and Pain Medicine, and Anesthesia and Pain Research Institute, Yonsei University College of Medicine, Seoul, Korea

A 50-year-old man weighing $48 \mathrm{~kg}$ was admitted to the hospital after suffering breathing difficulty and hoarseness for two weeks. During endoscopic examination, a squishy mass that occupied $80-90 \%$ of the glottic opening was observed (Fig. 1). The stalk was located on the left side just above the vocal cords and it moved slightly in and out of the glottis when the patient breathed. The patient entered the operating room in a sitting po-

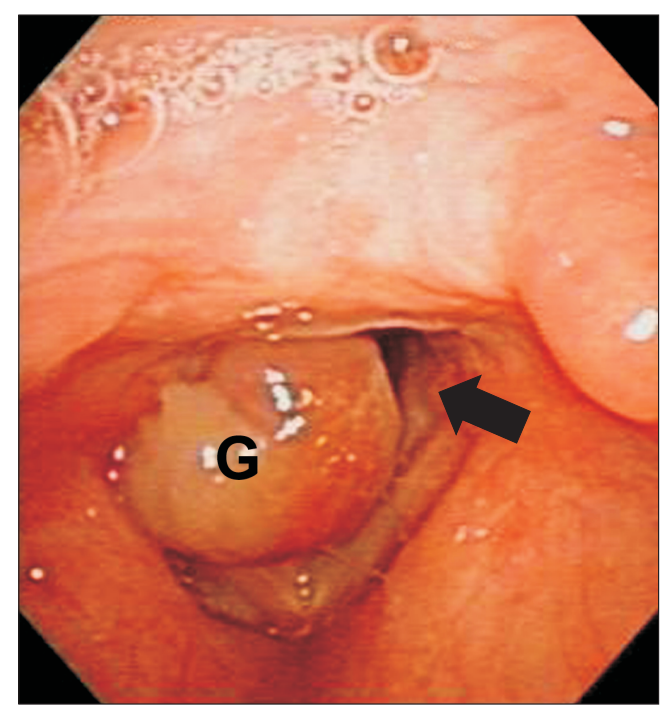

Fig. 1. An endoscopic view of the $3 \times 3 \mathrm{~cm}$-sized vocal cord granuloma. The arrow indicates a narrow glottic opening. G: granuloma. Used with permission from the patient. sition and was given $100 \%$ oxygen with a nasal prong. Once on the operating table, the patient was placed in the semi-Fowler's position. It was anticipated that when he lost consciousness from anesthesia induction, mask ventilation would no longer work due to his totally obstructed airway. We therefore decided to perform transtracheal jet ventilation (TTJV) using a percutaneous transtracheal catheter.

Intraoperative monitoring devices, such as an electrocardiogram, an automatic blood pressure cuff, and a pulse oximeter, were applied. Radial arterial cannulation was performed. The initial oxygen saturation was $100 \%$ in $100 \%$ oxygen and all other measured vital signs (BP: 147/91 mmHg, PR: 69 /min, RR: 15 / min) were within normal ranges. While in the semi-Fowler's position, the patient's neck was extended and, after skin infiltration with $1 \%$ lidocaine, a 16 gauge angiocatheter was inserted caudally at a $30^{\circ}$ angle via the cricothyroid membrane. After the needle was taken out, air was entering the catheter efficiently but was not aspirating well. We suspected that the catheter was touching the rear wall of the trachea, so approximately $1 \mathrm{~cm}$ was pulled out. Two $\mathrm{ml}$ of $4 \%$ lidocaine was injected through the catheter to anesthetize the tracheal lumen.

After intravenous injection of $0.1 \mathrm{mg}$ of glycopyrrolate, total intravenous anesthesia was performed using propofol and remifentanil. Manual ventilation with a facemask was not possible after loss of consciousness. Ventilation was difficult, but was maintained somewhat after immediate injection of $100 \mathrm{mg}$ of succinylcholine. As observed through direct laryngoscopy, the

Corresponding author: Wyun Kon Park, M.D., Ph.D., Department of Anesthesiology and Pain Medicine, and Anesthesia and Pain Research Institute, Yonsei University College of Medicine, 50, Yonsei-ro, Seodaemun-gu, Seoul 120-752, Korea. Tel: 82-2-2227-4172, Fax: 82-2-312-7185, E-mail:wkp7ark@yuhs.ac

(c) This is an open-access article distributed under the terms of the Creative Commons Attribution Non-Commercial License (http:// creativecommons.org/licenses/by-nc/3.0/), which permits unrestricted non-commercial use, distribution, and reproduction in any medium, provided the original work is properly cited. 
glottic opening was almost entirely covered by the mass but a narrow opening was seen. We changed our plan to transglottal jet ventilation instead of transtracheal ventilation, and a hard and long catheter (ID: $2 \mathrm{~mm}$, OD: $3 \mathrm{~mm}$, length: $50 \mathrm{~cm}$ ) was inserted though the glottic opening. The catheter tip was placed in the midtrachea. After insertion, using a fine-time controllable jet ventilator [1,2], a driving gas pressure of 50 psi $(2,586$ $\mathrm{mmHg}$ ) and an injection time of $0.75 \mathrm{~s}$ were applied and ventilation efficacy was confirmed by watching the chest expand during inspiration and sink during expiration. After observing chest wall movement, an initial injection of $10 \mathrm{mg}$ of atracurium was administered intravenously and, thereafter, muscle relaxation was maintained with intermittent injections of atracurium. Via the suspension laryngoscope, a round-shaped granuloma measuring approximately $3 \times 3 \mathrm{~cm}$ was observed. Because the mass was soft and friable, it could not be extracted in one step, so it was removed piecemeal instead. To minimize interruptions to the extraction process, we reduced the respiration rate as low as possible while watching the patient's $\mathrm{SpO}_{2}$ level on the pulse oximeter. After removing all of the vocal fold granuloma, we found that the angiocatheter was located very close to the rear wall of the trachea.

After the operation, a conventional endotracheal tube (ID: $6.0 \mathrm{~mm}$ ) was inserted and mechanical ventilation was carried out. End-tidal $\mathrm{CO}_{2}\left(\mathrm{ETCO}_{2}\right)$ measured immediately after inser- tion of the endotracheal tube was $57 \mathrm{mmHg}$, indicating mild hypercarbia. $\mathrm{ETCO}_{2}$ normalized soon and muscle relaxation was reversed. The patient was then extubated and, simultaneously, the 16 gauge angiocatheter was removed from the neck. The operation lasted for $35 \mathrm{~min}$ and the patient was anesthetized for a total of $1 \mathrm{~h}$ and $10 \mathrm{~min}$. Six days after surgery, he was discharged without any adverse events.

During jet ventilation, expiration is entirely passive and deflation occurs by spontaneous recoil of the chest and lungs when oxygen flow is stopped. Due to the need for a partially open airway to allow for passive exhalation [3], the prerequisite for TTJV is that there should be adequate expiratory flow. The subatmospheric pressure generated by the jet just below the larynx would force the mass toward the glottic opening, resulting in total airway obstruction. However, even with a massive laryngeal tumor causing severe inspiratory obstruction, adequate expiratory flow is possible because of the "ball-valve" effect of most laryngeal masses [4]. Immediately after jet injection, we observed that the mass and some blood were discharged out of the glottis. Although the Venturi principle may produce a small suction effect at the start of the inspiratory phase, this is rapidly negated by an upward flow of gas through the larynx as pressure rises high in the trachea after injection. The high intra-tracheal pressure caused by the jet ventilation appears to lift the mass up and open the obstructed glottis, allowing for better visualization [5].

\section{References}

1. Kil HK, Park WK, Roh JH, Shim JK, Kim HD, Ahn JH, et al. Development of fine time controller of a transtracheal jet ventilator and its experimental application. Korean J Anesthesiol 2002; 43: 93-100.

2. Kim HD, Ahn JH, Park WK, Kil HK, Kim DW. The development of an inspiratory time adjustable transtracheal jet ventilator and evaluation in a human trachea-lung model. Korean J Anesthesiol 2001; 40: 211-9.

3. Smith RB, Schaer WB, Pfaeffle H. Percutaneous trantracheal ventilation for anaesthesia and resuscitation: a review and report of complications. Can Anaesth Soc J 1975; 22: 607-12.

4. Weymuller EA Jr, Pavlin EG, Paugh D, Cummings CW. Management of difficult airway problems with percutaneous transtracheal ventilation. Ann Otol Rhinol Laryngol 1987; 96: 34-7.

5. Chandradeva K, Palin C, Ghosh SM, Pinches SC. Percutaneous transtracheal jet ventilation as a guide to tracheal intubation in severe upper airway obstruction from supraglottic oedema. Br J Anaesth 2005; 94: 683-6. 